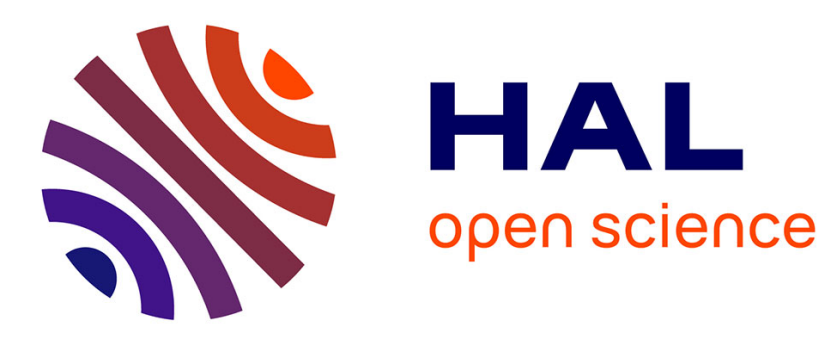

\title{
On the modelling of complex anisotropic frictional contact laws
}

Mohammed Hjiaj, Zhi-Qiang Feng, Géry de Saxcé, Zenon Mróz

\section{To cite this version:}

Mohammed Hjiaj, Zhi-Qiang Feng, Géry de Saxcé, Zenon Mróz. On the modelling of complex anisotropic frictional contact laws. International Journal of Engineering Science, 2004, 42 (10), pp.1013-1034. 10.1016/j.ijengsci.2003.10.004 . hal-01179518

\section{HAL Id: hal-01179518 \\ https://hal.science/hal-01179518}

Submitted on 2 Jul 2018

HAL is a multi-disciplinary open access archive for the deposit and dissemination of scientific research documents, whether they are published or not. The documents may come from teaching and research institutions in France or abroad, or from public or private research centers.
L'archive ouverte pluridisciplinaire HAL, est destinée au dépôt et à la diffusion de documents scientifiques de niveau recherche, publiés ou non, émanant des établissements d'enseignement et de recherche français ou étrangers, des laboratoires publics ou privés. 


\title{
On the modelling of complex anisotropic frictional contact laws
}

\author{
M. Hjiaj ${ }^{\text {a,*,1, Z.-Q. Feng }}{ }^{\text {b }}$, G. de Saxcé ${ }^{\mathrm{c}}$, Z. Mróz ${ }^{\mathrm{d}}$ \\ a Department of Civil Engineering, The University of Newcastle, University Drive, Callaghan, \\ NSW 2308, Australia \\ ${ }^{\mathrm{b}}$ Laboratoire de Mécanique d'Evry, Université d'Evry-Val d'Essonne, 40 rue du Pelvoux, 91020 Evry Cedex, France \\ ${ }^{\mathrm{c}}$ Laboratoire de Mécanique de Lille CNRS/UMR 8107, Boulevard Paul Langevin, \\ F-59655 Villeneuve d'Ascq Cedex, France \\ d Institute of Fundamental Technological Research, Polish Academy of Sciences, Świętokrzyska 21, \\ 00-049 Warsaw, Poland
}

In this paper, the formulation of complex anisotropic frictional models with orthotropic friction condition and non-associated sliding rule is discussed. The friction law is described by a superellipse, which allow to consider a wide range of convex friction condition by simply varying the roundness factor affecting the shape of the limit surface. The sliding potential is also a superellipse but with a different semi-axis ratio, which lead to a non-associated sliding rule. For bodies in contact, the Signorini conditions can be formulated in terms of velocities and combined with the sliding rule to give the frictional contact law describing interfacial interactions. Its is shown that the frictional contact law as well as its inverse can be derived from the same scalar valued function called bi-potential. Due to the non-associated nature of the law, this relation is implicit. The advantage of the present formulation lies in the existence of stationary points of a functional, called bi-functional, that depends on both the displacements and the stresses.

Keywords: Superelliptic friction criterion; Non-associated sliding rule; Bi-potential; Variational formulation

\footnotetext{
${ }^{*}$ Corresponding author. Tel.: +61-2-4921-5582; fax: +61-2-4921-6991.

E-mail address: mohammed.hjiaj@newcastle.edu.au (M. Hjiaj).

${ }^{1}$ Supported by the Australian Research Council.
} 


\section{Introduction}

Contact problems involving friction are of crucial importance in several engineering branches because the external loads are almost always applied to a deformable body via a contact surface; the other ways of loading being by fluid pressure (on the boundary) and by gravity (body forces). Moreover, the reaction to maintain the bodies in equilibrium will be almost invariably provided at a contact interface. Real contacting surface are frictional and the distribution of the surface traction on the contact zone is of considerable importance in a design process. Indeed, contact usually generate stress concentrations in the vicinity of the contact area which is therefore likely a site for material failure. If friction exists on the contact surface, in addition to normal contact forces, friction forces appear on the contact plane that resist sliding. In engineering applications such as wear, lubrication and rolling to name a few, an accurate description of the frictional behavior on the contact surface is required. This has stimulated the development of realistic mathematical models of friction. These models are rather complex and their implementation in numerical tools (FEM codes) are still a challenge.

A friction model is completely defined by the friction condition which specify a set of admissible contact forces and the sliding rule which stipulates what directions of sliding are allowed. The limit surface is usually assumed to be isotropic predicting a frictional behavior independent of the sliding direction. For many industrial applications, this assumption seems to be unrealistic and many experimental studies show that the frictional behavior can change drastically with the sliding direction, requiring an anisotropic model. The origin of this anisotropy can be attributed to two different sources. The first one is the material itself. The anisotropies of the materials constituting the bodies manifest themselves on the contact surface as well. The second one is technological; the industrial process used to fabricate the bodies can create striations along preferential directions. In fact, most machining, finishing and superfinishing operations are directional, and machined surfaces have particular striation patterns unique to type of machining. Also specific techniques of manufacture produce a surface with anisotropic frictional properties. For a large number of machining processes, the striation directions are mutually orthogonal. For such surfaces, an orthotropic model will provide a better description of the frictional behavior.

In papers devoted to orthotropic frictional contact problems, an associated sliding rule in the contact plane is assumed. However there is no particular reason for preventing the sliding rule being non-associated. Furthermore, experimental evidence shows that the sliding rule can deviate significantly from the normal to the friction condition in the plane $r_{n}=$ const, where $r_{n}$ is the contact pressure. In the sequel, the term "contact pressure" means the normal component of the contact force distribution which has a tangential component when friction exists on the contact surface. The occurrence of non-associated sliding rules is supported by theoretical investigations carried out by Michałowski and Mróz [5]. They considered a model of rigid anisotropic asperities, and proved that, in general, a non-associated sliding rule occurs within the contact plane with a possible concavity of the limit friction surface. In their study, they suggested that a large class of orthotropic frictional behaviors can be modelled by considering elliptic limit surfaces. To represent non-associated sliding rule, an elliptic sliding potential is adopted but with a different semiaxes ratio. In a subsequent paper, Mróz and Stupkiewicz broadened the class of orthotropic frictional models by considering superelliptic frictions conditions. The sliding potential is also a superellipse and a non-associated slip rule is obtained by choosing a different semi-axes ratio and 
(or) a different roundness parameter that controls the shape of the superellipse. With these two degrees of freedom, it is possible to deal with almost all orthotropic behaviors and therefore this modelling is very general.

The aim of this paper is to present a unified formulation of complex interface laws with superelliptic friction condition and non-associated sliding rule based on a variational statement of the law using the bi-potential approach. The present paper amounts to generalize previous work [2,3] on isotropic Coulomb's frictional contact law to superelliptic (anisotropic) friction conditions with non-associated sliding rule. For reasons, which will appear clear later, the present study is restricted to convex friction conditions. In the next section, contact variables are defined and the unilateral contact law is presented. For bodies in contact, this law can be written in a rate form where the kinematical variable is the normal velocity. Section 3 is concerned with anisotropic friction models with convex limit friction surfaces of superelliptic shape. A non-associated sliding rule is considered by adopting a different semi-axes ratio. At the end of Section 3, the rate form of the Signorini conditions are coupled with the sliding rule to give the complete frictional contact law for bodies in contact. In Section 4, variational formulations of the contact law, the frictional law and the frictional contact law are discussed in depth. The derivation of the bi-potential is given with details.

\section{Kinematic and static contact variables}

In order to formulate the frictional contact laws, appropriate kinematic and static variables need to be defined. For this reason the basics of continuum contact mechanics are briefly addressed in this section. The details are omitted as they are not necessary for the current study. Consider two deformable bodies $\mathscr{B}^{\alpha}$ (Fig. 1), $\alpha=1,2$, undergoing small displacement. Each of them occupies the open, simply connected, bounded domain $\Omega^{\alpha} \subset \mathbb{R}^{3}$, whose generic point is denoted $\mathbf{X}^{\alpha}$. The boundary $\Gamma^{\alpha}$ of each body is assumed to be sufficiently smooth everywhere such that an outward unit normal vector, denoted by $\mathbf{n}^{\alpha}$, can be defined at any point $M$ on $\Gamma^{\alpha}$. At each

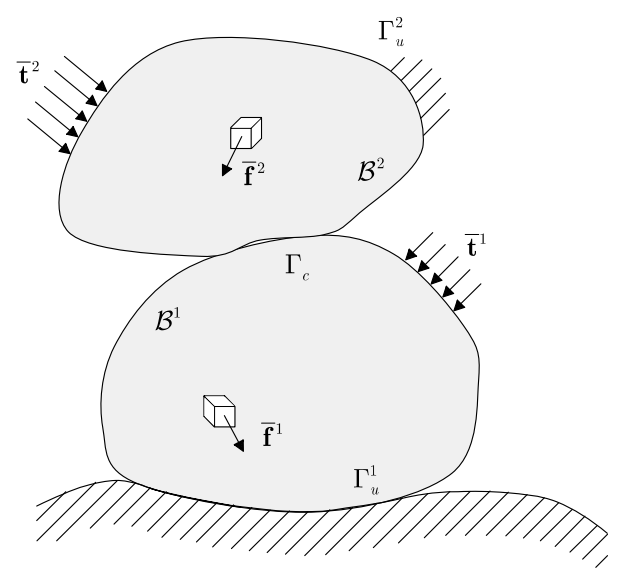

Fig. 1. Contact mechanics. 
time $t \in \mathbf{I}$, where $\mathbf{I}=[0, T]$ denotes the time domain of interest corresponding to the loading process, the boundary $\Gamma^{\alpha}$ of the body $\mathscr{B}^{\alpha}$ can, in general, be split into three parts: $\Gamma_{u}^{\alpha}$ with prescribed displacements, $\Gamma_{t}^{\alpha}$ with prescribed boundary loads, and the potential contact surface $\Gamma_{\mathrm{c}}$ where the two bodies $\mathscr{B}^{t}$ and $\mathscr{B}^{2}$ may possibly come into contact at some time $t$ :

$$
\Gamma^{\alpha}=\Gamma_{u}^{\alpha} \cup \Gamma_{t}^{\alpha} \cup \Gamma_{\mathrm{c}}^{\alpha}
$$

Although the two bodies may be in contact on several disjoint surface, we designate their union by a single symbol $\Gamma_{\mathrm{c}}$. The successive deformed configurations of $\mathscr{B}^{\alpha}$ are identified at each time $t$ by the displacement fields $\mathbf{u}^{\alpha}$ defined on $\bar{\Omega}^{\alpha}$, the closure of $\Omega^{\alpha}$. On the contact surface $\Gamma_{\mathrm{c}}$, a unique normal $\mathbf{n}$ directed towards $\mathscr{B}^{1}\left(\mathbf{n} \equiv \mathbf{n}^{2}\right)$ is considered (Fig. 2) and two unit vectors $\mathbf{t}_{x}$ and $\mathbf{t}_{y}$ are defined within the tangent plane $\mathbf{T}$, orthogonal to $\mathbf{n}$ in $\mathbb{R}^{3}$. The unit vectors $\mathbf{n}, \mathbf{t}_{x}$ and $\mathbf{t}_{y}$ form an orthonormal local basis and several options are possible for setting up the base vectors according to a particular choice of the unit tangent vectors. The description of the interactions occurring on $\Gamma_{\mathrm{c}}$ can be performed using the well-known master-slave concept which requires to introduce the relative velocity with respect to $\mathscr{B}^{2}$ (master) at the contact point $M$

$$
\dot{\mathbf{u}}(M)=\dot{\mathbf{u}}^{1}(M)-\dot{\mathbf{u}}^{2}(M)
$$

where $\dot{\mathbf{u}}^{1}$ and $\dot{\mathbf{u}}^{2}$ are the instantaneous velocities of $\mathscr{B}^{1}$ (salve) and $\mathscr{B}^{2}$, respectively, at this point. The relative velocity $\dot{\mathbf{u}}$, in short velocity, belongs to the three-dimensional vector space $\mathscr{V}$. Let $\mathbf{r}$ be the contact force distribution exerted on $\mathscr{B}^{1}$ at $M$ from $\mathscr{B}^{2}$. Accordingly, $\mathscr{B}^{2}$ is subjected to the traction vector $-\mathbf{r}$. The contact force $\mathbf{r}$ belongs to the three-dimensional vector space $\mathscr{F}$, dual of $\mathscr{V}$. In the local coordinate system defined by the tangential plane $\mathbf{T}$ and the normal $\mathbf{n}$, any variable $\dot{\mathbf{u}}$ or $\mathbf{r}$ may be uniquely decomposed into normal and tangential components according to

$$
\begin{array}{ll}
\dot{\mathbf{u}}=\dot{\mathbf{u}}_{t}+\dot{u}_{n} \mathbf{n} & \dot{\mathbf{u}}_{t} \in \mathbf{T}, \quad \dot{u}_{n} \in \mathbb{R} \\
\mathbf{r}=\mathbf{r}_{t}+r_{n} \mathbf{n} & \mathbf{r}_{t} \in \mathbf{T}, \quad r_{n} \in \mathbb{R}
\end{array}
$$

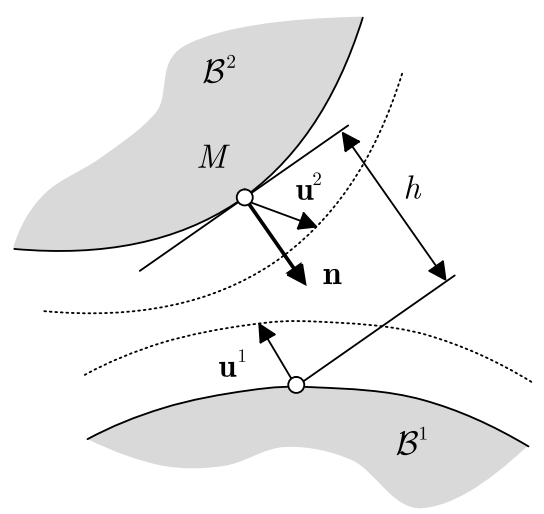

Fig. 2. Kinematics of contact. 


\subsection{Unilateral contact}

The unilateral contact condition impose that any point on the boundary of each body must either be in contact or not in contact. That is a body must not cross the boundary of the other one when they came into contact. This condition impose kinematical constraint on the displacement fields $\mathbf{u}^{1}$ and $\mathbf{u}^{2}$. Using the above decomposition, the non-penetration condition for a pair of bodies can be expressed by

$$
u_{n}+h \geqslant 0
$$

where $h$ is the initial gap:

$$
h=\left(\mathbf{X}^{1}\left(t_{0}\right)-\mathbf{X}^{2}\left(t_{0}\right)\right) \cdot \mathbf{n} \geqslant 0
$$

The dot "." in (3) represents the usual scalar product. A dual relation involves the contact pressure $r_{n}$ between the bodies which must be positive $\left(r_{n} \geqslant 0\right)$ where there is contact and zero where there is no contact. This condition is often referred to the non-adhesion condition. This set of relations may be summarized by the so-called Signorini conditions:

$$
u_{n}+h \geqslant 0, \quad r_{n} \geqslant 0, \quad\left(u_{n}+h\right) r_{n}=0
$$

which has to be satisfied at each time-instant $t \in \mathbf{I}$. These relations serve to determine which points will be in contact and which not. At any time $t \in \mathbf{I}$, the potential contact surfaces $\Gamma_{\mathrm{c}}^{\alpha}$ can be split into two disjoint parts: ${ }^{+} \Gamma_{\mathrm{c}}$ where the bodies are already in contact and ${ }^{-} \Gamma_{\mathrm{c}}^{\alpha}$ where the body are not in contact:

$$
\Gamma_{\mathrm{c}}^{\alpha}={ }^{+} \Gamma_{\mathrm{c}} \cup^{-} \Gamma_{\mathrm{c}}^{\alpha}
$$

In contrast to $\Gamma_{\mathrm{c}}^{\alpha}$ (problem data), ${ }^{+} \Gamma_{\mathrm{c}}$ and ${ }^{-} \Gamma_{\mathrm{c}}^{\alpha}$ change in time $t$ and can be empty at some $t \in \mathbf{I}$. On ${ }^{+} \Gamma_{\mathrm{c}}$, the unilateral contact conditions turns into

$$
u_{n} \geqslant 0, \quad r_{n} \geqslant 0, \quad u_{n} r_{n}=0 \quad \text { on }^{+} \Gamma_{\mathrm{c}}
$$

Moreover, the unilateral contact law can be formulated in a rate form:

$$
\dot{u}_{n} \geqslant 0, \quad r_{n} \geqslant 0, \quad \dot{u}_{n} r_{n}=0 \quad \text { on }^{+} \Gamma_{\mathrm{c}}
$$

The impenetrability condition (5a) expresses the fact that when the two bodies are in contact, then they must either remain in contact $\left(\dot{u}_{n}=0\right)$ or they must separate $\left(\dot{u}_{n}>0\right)$. We must stress that for this formulation only a loss of contact on ${ }^{+} \Gamma_{\mathrm{c}}$ is allowed and the extension of the contact area cannot be modelled with these relations. The rate formulation of the Signorini conditions (5) can be combined with the sliding rule to derive the full frictional contact law applicable to material points of $\Gamma_{\mathrm{c}}$ where bodies are in contact. This complete law specifies possible velocities of these points such that impenetrability, non-adhesion and the sliding rule are satisfied. Obviously, for a strictly positive gap $\left(u_{n}>0\right)$, the normal relative velocity is arbitrary $\left(\dot{u}_{n} \in \mathbb{R}\right)$ and the normal 
reaction force is equal to zero $\left(r_{n}=0\right)$. Motions of bodies that are not in contact are arbitrary until contact is made.

\section{Complex orthotropic friction models with non-associated sliding rule}

A rate-independent friction model is considered where a linear dependence of the limit tangential force on the normal force holds. This corresponds to a Coulomb-type friction model. Based on a model of rigid anisotropic asperities, a theoretical investigation on friction limit surfaces and sliding rules has been carried out by Michałowski and Mróz [5]. The outcome of their study shows that the level curves of the friction condition may be slightly non-convex but could be accurately approximated by ellipses. The sliding potential is also described by an ellipse but with different semi-axes ratio. Therefore, the sliding rule is no longer associated with the limit friction condition in the plane $r_{n}=$ const as it occurs when the sliding potential coincide with the friction condition for a known contact pressure. In a subsequent study, Mróz and Stupkiewicz [7] considered a more general shape for the friction condition and the sliding potential by adopting a superellipse for both. A non-associated slip rule can be obtained by adopting different semi-axes ratio, by choosing a different roundness factor or a combination of both effects. Such complex models allow to cover a large number of cases encountered in practice. Here, as a particular case, a family of anisotropic friction models, described by a friction condition and a sliding potential of superelliptic shape (in the plane $r_{n}=$ const) with identical roundness factor but different semi-axes ratio, is considered. The principal axes of both ellipses coincide with the orthogonal orthotropy axes $x$ and $y$.

\subsection{Superelliptical friction condition}

The asperity model used by Mróz and Stupkiewicz [7] to study anisotropic frictional contact phenomenon generates limit friction curves in the plane $r_{n}=$ const that can be slightly non-convex but very close to superellipses. Here, we consider only convex friction conditions that have the following form:

$$
f\left(r_{t_{x}}, r_{t_{y}}, r_{n}\right)=\left\|\mathbf{r}_{t}\right\|_{q_{\mu}}-r_{n}=0
$$

where $\|\bullet\|_{q_{\mu}}$ denotes the superelliptic norm

$$
\left.\left\|\mathbf{r}_{t}\right\|_{q_{\mu}}=\left|\frac{r_{t_{x}}}{\mu_{x}}\right|^{q}+\left|\frac{r_{t_{y}}}{\mu_{y}}\right|^{q}\right)^{\frac{1}{q}}
$$

with $1 \leqslant q<\infty$. The curve (6) intersects the $x$-axis at $\mu_{x} r_{n}$ and $-\mu_{x} r_{n}$; it intersects the $y$-axis at $\mu_{y} r_{n}$ and $-\mu_{y} r_{n}$ where the coefficients $\mu_{x}$ and $\mu_{y}$ are the principal friction coefficients. The coefficient $q$, called roundness factor, controls the shape of the friction criterion in the plane $r_{n}=$ const. This shape evolves from a parallelogram $(q=1)$ to a rectangle $(q \rightarrow \infty)$. The limit curves becomes a pinched diamond for $(q<1)$ and is no longer convex. When $q$ goes over 2, the curve become a 


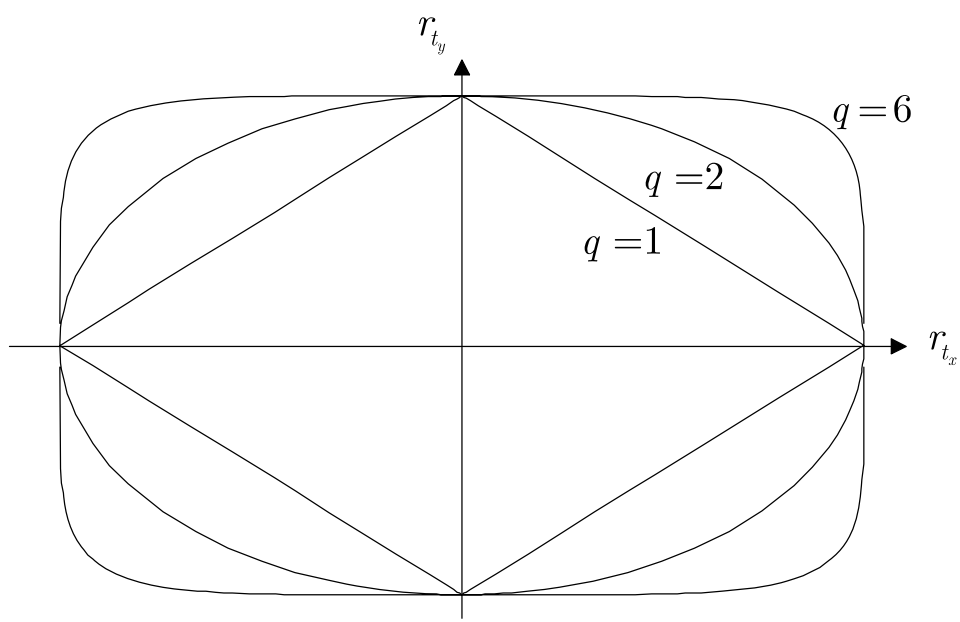

Fig. 3. Superelliptic friction condition.

rounded rectangle. Fig. 3 shows the limit friction condition for $q=1,2$ and 6 . The superellipse (7) can be described in a parametric form by:

$$
\left\{\begin{array}{l}
r_{t_{x}}= \pm \mu_{x} r_{n}|\cos \theta|^{\frac{2}{q}} \\
r_{t_{y}}= \pm \mu_{y} r_{n}|\sin \theta|^{\frac{2}{q}}
\end{array} \quad-\pi \leqslant \theta \leqslant \pi\right.
$$

By introducing the friction coefficients matrix

$$
\mathbb{M}=\left[\begin{array}{cc}
\mu_{x} & 0 \\
0 & \mu_{y}
\end{array}\right]
$$

the anisotropic superelliptic norm (7) is equivalent to the superelliptic norm $\|\bullet\|_{q}$ by

$$
\left\|\mathbf{r}_{t}\right\|_{q_{\mu}}=\left\|\mathbb{M}^{-1} \mathbf{r}_{t}\right\|_{q}
$$

The classical isotropic Coulomb's friction condition is recovered by setting

$$
\mu_{x}=\mu_{y}=\mu \text { and } q=2
$$

The set of allowable contact forces $K_{\mu}$ defined by

$$
K_{\mu}=\left\{\mathbf{r} \in \mathbb{R}^{3} \mid\left\|\mathbf{r}_{t}\right\|_{q_{\mu}}-r_{n} \leqslant 0\right\}
$$

is convex. The boundary and the interior of $K_{\mu}$ are denoted "bd $K_{\mu}$ " and "int $K_{\mu}$ ", respectively. The polar cone is defined by

$$
K_{\mu}^{*}=\left\{\mathbf{v} \in \mathbb{R}^{3} \mid\left\|\mathbf{v}_{t}\right\|_{q_{\mu}}^{*}+v_{n} \leqslant 0\right\}
$$

where the norm $\|\bullet\|_{q_{\mu}}^{*}$, dual of $\|\bullet\|_{q_{\mu}}$, is given by 


$$
\left\|\mathbf{v}_{t}\right\|_{q_{\mu}}^{*}=\left(\left|\mu_{x} v_{t_{x}}\right|^{s}+\left|\mu_{y} v_{t_{y}}\right|^{s}\right)^{\frac{1}{s}}=\left\|\mathbb{M} \mathbf{v}_{t}\right\|_{q}^{*}
$$

The coefficients $q$ and $s$ satisfy

$$
\frac{1}{q}+\frac{1}{s}=1
$$

Any pair $(\mathbf{r}, \mathbf{v})$ such that $\mathbf{r} \in K_{\mu}$ and $\mathbf{v} \in K_{\mu}^{*}$ satisfies

$$
\mathbf{r} \cdot \mathbf{v} \leqslant 0
$$

\subsection{Superelliptical sliding potentials}

The frictional force are dissipative as they always oppose the sliding velocity. To emphasize this physical aspect of the law, the relative velocity will be preceded by a sign "minus" $(-\dot{\mathbf{u}})$. Therefore its tangential component $\left(-\dot{\mathbf{u}}_{t}\right)$ can be represented, in the force space, by an outward normal vector with respect to level curves of the friction cone (associated slip rule). The convex slip potential has also superelliptic level curves with identical roundness factor but with different semiaxes ratio (Fig. 4). The generic expression of the sliding potential is

$$
g\left(r_{t_{x}}, r_{t_{y}}\right)=\left\|\mathbf{r}_{t}\right\|_{q_{p}}-a=0
$$

in which $\left\|\mathbf{r}_{t}\right\|_{q_{p}}$ is given by

$$
\left\|\mathbf{r}_{t}\right\|_{q_{p}}=\left(\left|\frac{r_{t_{x}}}{p_{x}}\right|^{q}+\left|\frac{r_{t_{y}}}{p_{y}}\right|^{q}\right)^{\frac{1}{q}}=\left\|\mathbb{P}^{-1} \mathbf{r}_{t}\right\|_{q}
$$

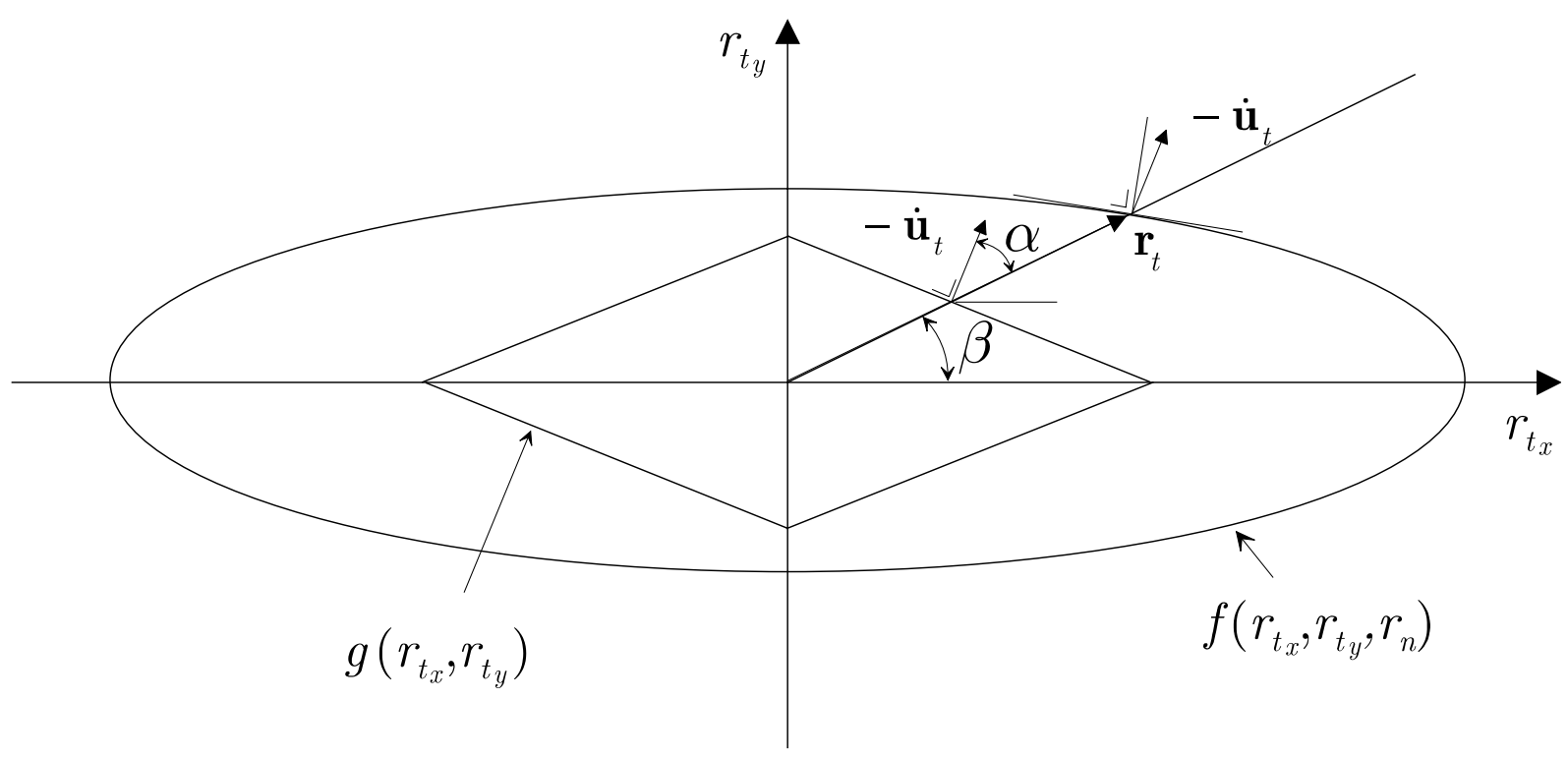

Fig. 4. Superelliptic sliding potential. 
with

$$
\mathbb{P}=\left[\begin{array}{cc}
p_{x} & 0 \\
0 & p_{y}
\end{array}\right]
$$

In the definition of the sliding potential (12), $a$ is a positive constant whose magnitude is irrelevant. The semi-axes ratio of the slip potential is related to the one of the friction condition by the following relation

$$
\frac{p_{y}}{p_{x}}=\left(\frac{\mu_{y}}{\mu_{x}}\right)^{k}
$$

In the general case, we have $k \neq 1$, which leads to a non-associated sliding rule

$$
\begin{aligned}
& -\dot{u}_{n}=0 \\
& -\dot{u}_{t_{x}}=\dot{\lambda} \frac{\partial g}{\partial r_{t_{x}}}=\dot{\lambda}\left(\left|\frac{r_{t_{x}}}{p_{x}}\right|^{q}+\left|\frac{r_{t_{y}}}{p_{y}}\right|^{q}\right)^{\frac{1-q}{q}} \frac{1}{p_{x}^{q}}\left|r_{t_{x}}\right|^{q-1} \operatorname{sgn}\left(r_{t_{x}}\right) \\
& -\dot{u}_{t_{y}}=\dot{\lambda} \frac{\partial g}{\partial r_{t_{y}}}=\dot{\lambda}\left(\left|\frac{r_{t_{x}}}{p_{x}}\right|^{q}+\left|\frac{r_{t_{y}}}{p_{y}}\right|^{q}\right)^{\frac{1-q}{q}} \frac{1}{p_{y}^{q}}\left|r_{t_{y}}\right|^{q-1} \operatorname{sgn}\left(r_{t_{y}}\right)
\end{aligned}
$$

where the multiplier $\dot{\lambda}$ is equal to

$$
\dot{\lambda}=\left(\left(p_{x}\left|-\dot{u}_{t_{x}}\right|\right)^{\frac{q}{q-1}}+\left(p_{y}\left|-\dot{u}_{t_{y}}\right|\right)^{\frac{q}{q-1}}\right)^{\frac{q-1}{q}}
$$

and the signum function $\operatorname{sgn}(\bullet)$ is defined by

$$
\operatorname{sgn}(x)= \begin{cases}1 & \text { for } x>0 \\ 0 & \text { for } x=0 \\ -1 & \text { for } x<0\end{cases}
$$

Taking into account (11) the expression (18) of the multiplier $\dot{\lambda}$ can be transformed into

$$
\dot{\lambda}=\left\|-\dot{\mathbf{u}}_{t}\right\|_{q_{p}}^{*}=\left(\left(p_{x}\left|-\dot{u}_{t_{x}}\right|\right)^{s}+\left(p_{y}\left|-\dot{u}_{t_{y}}\right|\right)^{s}\right)^{\frac{1}{s}}
$$

The sliding direction is given by the gradient to the potential and the magnitude by the multiplier $\dot{\lambda}$. Therefore, the expression between the parentheses in (16) and (17) can be modified by replacing $p_{\bullet}$ by $\mu_{\bullet}$ without violating the sliding rule. The multiplier $\dot{\lambda}$ is no longer the same and is denoted $\dot{\lambda}^{\prime}$. The sliding rule is now given by

$$
\begin{aligned}
& -\dot{u}_{n}=0 \\
& \left.-\dot{u}_{t_{x}}=\dot{\lambda}^{\prime}\left|\frac{r_{t_{x}}}{\mu_{x}}\right|^{q}+\left|\frac{r_{t_{y}}}{\mu_{y}}\right|^{q}\right)^{\frac{1-q}{q}} \frac{1}{p_{x}^{q}}\left|r_{t_{x}}\right|^{q-1} \operatorname{sgn}\left(r_{t_{x}}\right)
\end{aligned}
$$




$$
\left.-\dot{u}_{t_{y}}=\dot{\lambda}^{\prime}\left|\frac{r_{t_{x}}}{\mu_{x}}\right|^{q}+\left|\frac{r_{t_{y}}}{\mu_{y}}\right|^{q}\right)^{\frac{1-q}{q}} \frac{1}{p_{y}^{q}}\left|r_{t_{y}}\right|^{q-1} \operatorname{sgn}\left(r_{t_{y}}\right)
$$

where the multiplier $\dot{\lambda}^{\prime}$ is equal to

$$
\dot{\lambda}^{\prime}=\left\|\mathbb{Q}^{q}\left(-\dot{\mathbf{u}}_{t}\right)\right\|_{q_{\mu}}^{*}
$$

The matrix $\mathbb{Q}$ defined by

$$
\mathbb{Q}=\mathbb{P M}^{-1}=\left[\begin{array}{cc}
p_{x} / \mu_{x} & 0 \\
0 & p_{y} / \mu_{y}
\end{array}\right]=\mathbb{M}^{-1} \mathbb{P}
$$

is called the sliding non-associativity matrix. Obviously, relations exist between different norms. For instance, it holds

$$
\left\|\mathbb{Q}^{q-1}\left(-\dot{\mathbf{u}}_{t}\right)\right\|_{q_{p}}^{*}=\left\|\mathbb{Q}^{q}\left(-\dot{\mathbf{u}}_{t}\right)\right\|_{q_{\mu}}^{*}
$$

The inverse of the relationships (20)-(22) is

$$
\begin{aligned}
& r_{n}>0 \\
& \left|r_{t_{x}}\right|=r_{n} \frac{\left(p_{x}^{q}\left|-\dot{u}_{t_{x}}\right|\right)^{\frac{1}{q-1}}}{\left\|\mathbb{Q}^{q}\left(-\dot{\mathbf{u}}_{t}\right)\right\|_{q_{\mu}}^{*}} \\
& \left|r_{t_{y}}\right|=r_{n} \frac{\left(p_{y}^{q}\left|-\dot{u}_{t_{y}}\right|\right)^{\frac{1}{q-1}}}{\left\|\mathbb{Q}^{q}\left(-\dot{\mathbf{u}}_{t}\right)\right\|_{q_{\mu}}^{*}}
\end{aligned}
$$

Denoting the inclination of the force $\mathbf{r}_{t}$ to the $x$-axis by $\alpha$ and that of the velocity vector $\left(-\dot{\mathbf{u}}_{t}\right)$ by $\beta$, it follows from (25) and (26) that

$$
|\tan \alpha|=\frac{\left|r_{t_{y}}\right|}{\left|r_{t_{x}}\right|}=\left(\frac{p_{x}}{p_{y}}\right)^{\frac{q}{q-1}}|\tan \beta|^{\frac{1}{q-1}}
$$

The dissipation function is expressed as follows:

$$
D=r_{t_{x}}\left(-\dot{u}_{t_{x}}\right)+r_{t_{y}}\left(-\dot{u}_{t_{y}}\right)=\left\|\mathbf{r}_{t}\right\|_{q_{p}}\left\|\mathbb{Q}^{q-1}\left(-\dot{\mathbf{u}}_{t}\right)\right\|_{q_{p}}^{*}
$$

The present model is very general and is able to represent a large number of physical situations. By adequately adjusting the roundness factor and other parameters, many orthotropic frictional surface can be represented by such a model.

\subsection{Governing relations for an anisotropic frictional interface}

We consider now the previous friction law embedding an impenetrability condition for completeness. On the contact surface ${ }^{+} \Gamma_{\mathrm{c}}$, the sliding rule can be combined with a rate form of the 
unilateral contact conditions to obtain the frictional contact law. The multivalued nature of this strongly non-linear law makes problems involving frictional contact among the most difficult ones in solid mechanics. The complete form of the frictional contact law deals with the three possible physical situations, which are separation, contact with sticking, and contact with sliding. Only in the last situation, dissipation is produced. Two overlapped "if...then...else" statements can be used to write it analytically (Box 1):

Box 1

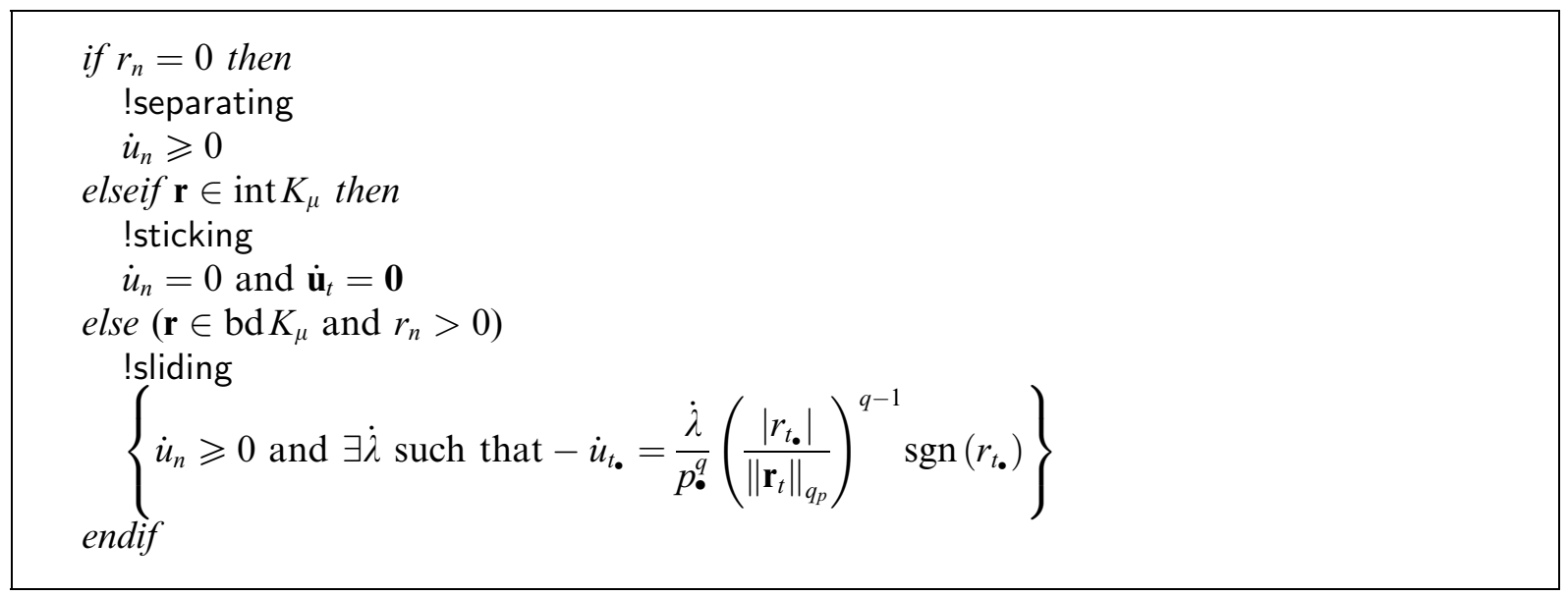

The multivalued character of the law comes into sight in the first and the second part of the statement. If $r_{n}$ is null then $\dot{\mathbf{u}}$ is arbitrary but its normal component $\dot{u}_{n}$ should be positive. In others words, one single element of $\mathscr{F}(\mathbf{r}=\mathbf{0})$ is associated with an infinite number of velocity vectors $\dot{\mathbf{u}} \in \mathscr{V}$. On the other hand, if $-\dot{\mathbf{u}}$ is null then $\mathbf{r}$ belongs to $K_{\mu}$ but can be arbitrary. So, one single element of the velocity space $\mathscr{V}(-\dot{\mathbf{u}}=\mathbf{0})$, is related to an infinite number of force vectors $\mathbf{r} \in \mathscr{F}$. The inverse law, i.e. the relationship $\mathbf{r}(-\dot{\mathbf{u}})$ can be written as (Box 2):

Box 2

if $\dot{u}_{n}>0$ then
$\quad$ !separating
$r_{n}=0$
elseif $\dot{\mathbf{u}}=\mathbf{0}$ then
$\quad$ !sticking
$\quad \mathbf{r} \in K_{\mu}$
else $(\dot{\mathbf{u}} \in \mathbf{T}-\{\mathbf{0}\})$
$\quad$ !sliding
$\quad\left\{\dot{u}_{n} \geqslant 0\right.$ and $\exists \dot{\lambda}$ such that $\left.r_{t_{\bullet}}=r_{n} \frac{\left(p_{\bullet}^{q}\left|-\dot{u}_{t_{\bullet}}\right|\right)^{\frac{1}{q-1}}}{\left\|\mathbb{Q}^{q-1}\left(-\dot{\mathbf{u}}_{t}\right)\right\|_{q_{p}}^{*}} \operatorname{sgn}\left(-\dot{u}_{t_{\bullet}}\right)\right\}$
endif




\section{Alternative formulations of the frictional contact law}

The previous forms of the frictional contact law are not very enlightening and a more instructive formulation is certainly welcome. Variational formulation are attractive because solution(s) of boundary value problems are stationary point(s) of a certain functional. Moreover, uniqueness of the solution is guaranteed if the functional is convex or concave. Existence of such functional in solid mechanics depends partly on the constitutive relationships and their mathematical structure. A necessary condition ensuring the existence of such functional is a "potential" structure of the relationship between dual variables. This mathematical structure exists for rateindependent model if the evolution law governing the dissipative process can be obtained via a normality rule. It is rather clear that this is not the case for the frictional contact model even if the sliding rule is associated. In fact, if we regard contact force $\mathbf{r}$ and velocity $-\dot{\mathbf{u}}$ as conjugate quantities of each other, the normality will not occur since it would require that the velocity would have a normal separating component. In what follows, we will present a variational formulation of the complex frictional contact model discussed in Section 3. Before that, we discuss first the unilateral contact law and recall the alternative existing formulations including the one based on a variational inequality and a differential inclusion. These formulations bear nice mathematical properties that can be exploited to study the solution properties of boundary value problem involving frictionless contact. Next, the sliding rule is presented under different forms. However, the variational formulations are not true one and are usually termed quasi-variational formulations. In these formulations, the contact pressure $r_{n}$ is taken as a parameter.

\subsection{Variational formulations of the unilateral contact law}

The graph of the unilateral contact law, shown in Fig. 5, is not the graph of a single-valued mapping since neither $-\dot{u}_{n}$ is a function of $r_{n}$ nor $r_{n}$ is a function of $-\dot{u}_{n}$. In fact, $r_{n}$ is positive but arbitrary for $-\dot{u}_{n}=0$ and is null for any positive value of $-\dot{u}_{n}$. This means that for one element of $\mathbb{R}_{+}$it corresponds the subset $\mathbb{R}_{-} \backslash\{0\}$ containing an infinite number of elements. Clearly, the relationship between $r_{n}$ and $-\dot{u}_{n}$ is multivalued. Therefore, a differentiable potential is not appropriate for this law. This is a typical example of a non-smooth relation. Accordingly, classical tools of differential calculus are not longer applicable. Instead, concept and tools of Convex Analysis [9] are used to deal with such law. It is easy to see that the relations (5) can be combined together to give the following variational inequality:

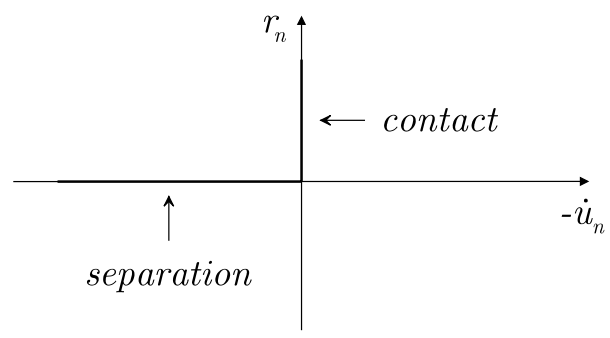

Fig. 5. Unilateral contact law. 


$$
r_{n} \in \mathbb{R}_{+}: \quad-\dot{u}_{n}\left(r_{n}-r_{n}^{\prime}\right) \geqslant 0, \quad \forall r_{n}^{\prime} \in \mathbb{R}_{+}
$$

Although the previous formulation possesses some interesting mathematical properties, it can be further rearranged to obtain the set-valued constitutive mapping expressing the relation between the contact pressure $r_{n}$ and the corresponding normal velocity $-\dot{u}_{n}$, i.e. the relation $-\dot{u}_{n}\left(r_{n}\right)$. The idea, due to Moreau [6], is to make use of the indicator function [9] of the set to which the contact pressures $r_{n}$ and $r_{n}^{\prime}$ are compelled to belong, i.e. $\mathbb{R}_{+}=[0,+\infty[$ :

$$
\Psi_{\mathbb{R}_{+}}\left(r_{n}\right)= \begin{cases}0 & \text { if } r_{n} \in \mathbb{R}_{+}, \\ +\infty & \text { otherwise }\end{cases}
$$

This function is not differentiable in the classical sense. However the indicator function is convex if the set to which it refers is convex. Having at hand this tool, a key-step is to rewrite the variational inequality (27) in the following manner

$$
-\dot{u}_{n}\left(r_{n}-r_{n}^{\prime}\right)+\Psi_{\mathbb{R}_{+}}\left(r_{n}^{\prime}\right) \geqslant \Psi_{\mathbb{R}_{+}}\left(r_{n}\right)
$$

where the member function " $\in$ " in (27) have been replaced by the value of the indicator function at the corresponding contact pressure. The inequality (29) corresponds to the convexity inequality applied to a non-differentiable function [9]. It means that $-\dot{u}_{n}$ belongs to the subdifferential of $\Psi_{\mathbb{R}_{+}}$ at $r_{n}$ or equivalently that $-\dot{u}_{n}$ and $r_{n}$ are related by the differential inclusion:

$$
-\dot{u}_{n} \in \partial \Psi_{\mathbb{R}_{+}}\left(r_{n}\right)
$$

The subdifferential of $\Psi_{\mathbb{R}_{+}}$at $r_{n}$ corresponds to the set of all subgradients of $\Psi_{\mathbb{R}_{+}}$at $r_{n}$ and defines a multivalued mapping from $\mathbb{R}$ into itself:

$$
\partial \Psi_{\mathbb{R}_{+}}: \mathbb{R} \mapsto \mathbb{R}: r_{n} \mapsto \partial \Psi_{\mathbb{R}_{+}}\left(r_{n}\right)
$$

In particular, for a differentiable function, the subdifferential is reduced to a singleton which corresponds to the classical gradient. The function $\Psi_{\mathbb{R}_{+}}\left(r_{n}\right)$ is called complementary contact pseudo-potential and denoted by $\psi_{\mathrm{C}}^{*}\left(r_{n}\right)$. The term "pseudo" is used to make a distinction between the classical differentiable potential like the one existing in Hookean elasticity and the present non-differentiable potential. The above considerations show that by simply allowing the potential to be non-differentiable, a potential structure of the relationship between $-\dot{u}_{n}$ and $r_{n}$ can be found. Accordingly, differentiable potentials are suited only for single-valued law and non-differentiable potentials provide an effective means to represent multivalued constitutive laws. With the setting (30), the law may be inverted by applying the Fenchel transform

$$
\psi_{\mathrm{C}}\left(-\dot{u}_{n}\right)=\sup _{r_{n}}\left[-\dot{u}_{n} r_{n}-\psi_{\mathrm{C}}^{*}\left(r_{n}\right)\right]=\Psi_{\mathbb{R}_{-}}\left(-\dot{u}_{n}\right)
$$

where $\mathbb{R}_{-}$corresponds to $\left.]-\infty, 0\right]$ and $\psi_{\mathrm{C}}$ is the contact pseudo-potential. The inverse contact law is then 


$$
r_{n} \in \partial \psi_{\mathrm{C}}\left(-\dot{u}_{n}\right)
$$

which is equivalent to

$$
r_{n}\left(\left(-\dot{u}_{n}\right)-\left(-\dot{u}_{n}^{\prime}\right)\right)+\Psi_{\mathbb{R}_{-}}\left(-\dot{u}_{n}^{\prime}\right) \geqslant \Psi_{\mathbb{R}_{-}}\left(-\dot{u}_{n}\right)
$$

It results from the previous inequality that

$$
\left(-\dot{u}_{n}\right) \in \mathbb{R}_{-}: \quad r_{n}\left(\left(-\dot{u}_{n}\right)-\left(-\dot{u}_{n}^{\prime}\right)\right) \geqslant 0, \quad \forall\left(-\dot{u}_{n}^{\prime}\right) \in \mathbb{R}_{-}
$$

which is the dual of (27). The formulations (27) and (31) of the unilateral contact law are particularly useful for associating dual extremum principles to boundary value problems involving frictionless contact.

\subsection{Variational formulations of the sliding rule}

Sliding can occur only if the bodies are in contact and the friction force attain a certain threshold proportional to the contact pressure. The graph of 2D Coulomb's law is displayed Fig. 4. This graph is "infinitely steep" and for that reason it can not be the graph of single-valued mapping. Because $r_{n}$ may take any arbitrary positive value, the graph should be considered as a family of monotone multivalued mappings. Traditional variational formulations of the sliding rule are well-known for the Coulomb criterion [6]. However, these formulations are not discussed in the literature for superelliptic friction condition. In this paragraph, earlier variational formulations of associated slip rule are extended to friction condition with a superelliptic shape in the plane $r_{n}=$ const. Non-associated sliding rules will be considered in the next section.

If we consider the normal reaction $r_{n}$ as a parameter (or state variable), the friction condition (6) becomes a function of the friction force $\mathbf{r}_{t}$ only and the slip law can be expressed under the form of a quasi-variational inequality [1]:

$$
\left\{\begin{array}{l}
\mathbf{r}_{t} \in \tilde{K}_{\mu}: \\
\left(-\dot{\mathbf{u}}_{t}\right) \cdot\left(\mathbf{r}_{t}^{\prime}-\mathbf{r}_{t}\right) \geqslant 0, \quad \forall \mathbf{r}_{t}^{\prime} \in \tilde{K}_{\mu}
\end{array}\right.
$$

where $\tilde{K}_{\mu}$ defined by

$$
\tilde{K}_{\mu}=\left\{\mathbf{r}_{t} \in \mathbb{R}^{2} \mid\left\|\mathbf{r}_{t}\right\|_{q_{\mu}} \leqslant k\right\} \quad \text { with } k=r_{n}
$$

is the section of Coulomb's cone at level $r_{n}$. The term "quasi" is used to emphasize the dependence of $\tilde{K}_{\mu}$ on $r_{n}$. The previous inequality suggests that the friction law can be formulated in the form of the principle of maximum dissipation: the actual friction force is an optimal solution for the maximization problem in the variable $\mathbf{r}_{t}$ :

$$
\begin{cases}\operatorname{maximize} & \left(-\dot{\mathbf{u}}_{t}\right) \cdot \mathbf{r}_{t} \\ \text { subject to } & \mathbf{r}_{t} \in \tilde{K}_{\mu}\end{cases}
$$


To further transform inequality (32) in the same way it has been done for the unilateral contact law, we introduce the indicator function of the closed convex set $\tilde{K}_{\mu}$ defined by

$$
\Psi_{\tilde{K}_{\mu}}\left(\mathbf{r}_{t}\right)= \begin{cases}0 & \text { if } \mathbf{r}_{t} \in \tilde{K}_{\mu}, \\ +\infty & \text { otherwise }\end{cases}
$$

This function is subsequently used to enforce the constraints on the friction force:

$$
\Psi_{\tilde{K}_{\mu}}\left(\mathbf{r}_{t}^{\prime}\right) \geqslant\left(-\dot{\mathbf{u}}_{t}\right) \cdot\left(\mathbf{r}_{t}^{\prime}-\mathbf{r}_{t}\right)+\Psi_{\tilde{K}_{\mu}}\left(\mathbf{r}_{t}\right)
$$

The inequality (34) corresponds to convexity condition applied to $\Psi_{\tilde{K}_{\mu}}$. Therefore, it means that $\left(-\dot{\mathbf{u}}_{t}\right)$ belongs to the subdifferential of $\Psi_{\tilde{K}_{\mu}}\left(\mathbf{r}_{t}\right)$ :

$$
-\dot{\mathbf{u}}_{t} \in \partial \Psi_{\tilde{K}_{\mu}}\left(\mathbf{r}_{t}\right)
$$

The indicator function $\Psi_{\tilde{K}_{\mu}}$ corresponds to the complementary frictional dissipation function, denoted by $\psi_{\mathrm{F}}^{*}$. Because of its dependance on $r_{n}, \psi_{\mathrm{F}}^{*}$ is a quasi-pseudo-potential. Again, $\partial \Psi_{\tilde{K}_{\mu}}\left(\mathbf{r}_{t}\right)$ is a set-valued mapping and the relation (35) coincide with sliding rule. To inverse the slip rule, the Fenchel transform is applied:

$$
\psi_{\mathrm{F}}\left(-\dot{\mathbf{u}}_{t}\right)=\sup _{\mathbf{r}_{t}}\left[\left(-\dot{\mathbf{u}}_{t}\right) \cdot \mathbf{r}_{t}-\Psi_{\tilde{K}_{\mu}}\left(\mathbf{r}_{t}\right)\right]=\sup _{\mathbf{r}_{t} \in \tilde{K}_{\mu}}\left[\left(-\dot{\mathbf{u}}_{t}\right) \cdot \mathbf{r}_{t}\right]
$$

The scalar product in (36) represent the dissipation and satisfies the following inequalities:

$$
-\dot{u}_{t_{x}} r_{t_{x}}+-\dot{u}_{t_{y}} r_{t_{y}} \leqslant\left|-\dot{u}_{t_{x}}\right|\left|r_{t_{x}}\right|+\left|-\dot{u}_{t_{y}}\right|\left|r_{t_{y}}\right| \leqslant\left|\mu_{x}\left(-\dot{u}_{t_{x}}\right)\right|\left|\frac{r_{t_{x}}}{\mu_{x}}\right|+\left|\mu_{y}\left(-\dot{u}_{t_{y}}\right)\right|\left|\frac{r_{t_{y}}}{\mu_{y}}\right| \leqslant\left\|\mathbf{r}_{t}\right\|_{q_{\mu}}\left\|\left(-\dot{\mathbf{u}}_{t}\right)\right\|_{q_{\mu}}^{*}
$$

where the Hölder inequality (see Appendix A) has been used in the last step. Using the friction condition, the dissipation is bounded by

$$
-\dot{u}_{t_{x}} r_{t_{x}}+-\dot{u}_{t_{y}} r_{t_{y}} \leqslant r_{n}\left\|\left(-\dot{\mathbf{u}}_{t}\right)\right\|_{q_{\mu}}^{*}
$$

Therefore, the frictional dissipation quasi-pseudo-potential is given by

$$
\psi_{\mathrm{F}}\left(-\dot{\mathbf{u}}_{t}\right)=r_{n}\left\|\left(-\dot{\mathbf{u}}_{t}\right)\right\|_{q_{\mu}}^{*}
$$

where the dependance of $\psi_{\mathrm{F}}$ on $r_{n}$ is regarded as parametric. This pseudo-potential provides the dissipation for a known contact pressure $r_{n}$ and the inverse sliding rule is given by

$$
\mathbf{r}_{t} \in \partial \psi_{\mathrm{F}}\left(-\dot{\mathbf{u}}_{t}\right)
$$

The quasi-pseudo-potential is a convex (by construction) and a positively homogenous function of order one with respect to $-\dot{\mathbf{u}}_{t}$. It does not exist anymore if the sliding rule is non-associated. The two quasi-pseudo-potentials are related by the Fenchel inequality 


$$
\psi_{\mathrm{F}}\left(-\dot{\mathbf{u}}_{t}^{\prime}\right)+\psi_{\mathrm{F}}^{*}\left(\mathbf{r}_{t}^{\prime}\right) \geqslant\left(-\dot{\mathbf{u}}_{t}^{\prime}\right) \cdot \mathbf{r}_{t}^{\prime} \quad \forall\left(\left(-\dot{\mathbf{u}}_{t}^{\prime}\right), \mathbf{r}_{t}^{\prime}\right) \in \mathscr{V} \times \mathscr{F}
$$

The equality is reached for a pair $\left(-\dot{\mathbf{u}}_{t}, \mathbf{r}_{t}\right)$ satisfying the sliding rule

$$
\psi_{\mathrm{F}}\left(-\dot{\mathbf{u}}_{t}\right)+\psi_{\mathrm{F}}^{*}\left(\mathbf{r}_{t}\right)=\left(-\dot{\mathbf{u}}_{t}\right) \cdot \mathbf{r}_{t}
$$

The work of Moreau [6] on the mathematical structure of mechanical laws is an important step in material modeling. The main contribution is probably the unified framework proposed for mechanical laws including the multivalued ones. The chief idea is to consider non-differentiable potential if the constitutive relation is multivalued. To properly deal with such functions, Moreau used concepts and tools of Convex Analysis. Particularly, the notion of indicator function has been proven fruitful to the treatment of rate-independent model. The corresponding formulation and its variational structure revealed by the "potential form" of constitutive relationship prove to be useful regarding to the numerical and mathematical aspects of boundary value problems. This property ensures the existence of stationary principles that becomes minimum principles if the functional is convex. We recall that "existence of a potential" and "convexity" are two separate matters. We can say on one hand that a single-valued law can be derived from a differentiable potential if this one really exists. On the other hand, a multivalued law can be derived from a nondifferentiable potential under the assumption that it exists. The non-differentiability precisely reflects the multivalued character of the physical law. Another key-step has been accomplished by Nguyen Quoc Son [4,8] who extends Moreau's work to more complex multivalued laws ('visco'plasticity with hardening, damage,...) using the phenomenological approach with internal variables. Although the introduction of the pseudo-potential concept is an important step in the formulation of the behavior of materials, some models cannot fall within the framework. Probably, the frictional contact law is the most familiar among such models.

\subsection{Variational formulations of the frictional contact law}

\subsubsection{Associated sliding rule}

The sliding rule bears some resemblance with the flow rule in plasticity. This analogy can be further extended to the frictional contact law for bodies in contact. Let us examine Fig. 6

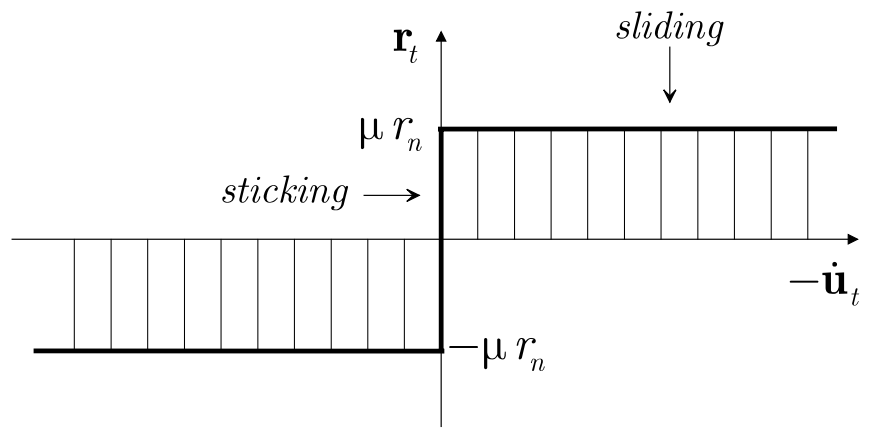

Fig. 6. Friction law. 
(isotropic friction criterion) and assume that the sliding rule is associated. For the sliding status, the normality rule can be recovered by adding " $\mu\left\|-\dot{\mathbf{u}}_{t}\right\| \mathbf{n}$ " to normal to the Coulomb's cone:

$$
-\dot{\mathbf{u}}_{t} \in \partial \Psi_{K_{\mu}}(\mathbf{r})+\mu\left\|-\dot{\mathbf{u}}_{t}\right\| \mathbf{n}
$$

The friction cone being differentiable everywhere except at the origin $\left(r_{n}=0\right)$, the previous relation is equivalent to

$$
-\dot{\mathbf{u}}_{t}=\dot{\lambda} \frac{\partial f}{\partial \mathbf{r}}+\mu\left\|-\dot{\mathbf{u}}_{t}\right\| \mathbf{n}
$$

for a non-null contact pressure. Since during sliding, we have $\dot{u}_{n}=0$, we can add $\dot{u}_{n}$ to the last term

$$
-\dot{\mathbf{u}}_{t}=\dot{\lambda} \frac{\partial f}{\partial \mathbf{r}}+\left(\dot{u}_{n}+\mu\left\|-\dot{\mathbf{u}}_{t}\right\|\right) \mathbf{n}
$$

without violating the sliding rule. By rearranging (41), we find that the sliding rule takes the following form

$$
-\left(\dot{\mathbf{u}}_{t}+\left(\dot{u}_{n}+\mu\left\|-\dot{\mathbf{u}}_{t}\right\|\right) \mathbf{n}\right) \in \partial \Psi_{K_{\mu}}(\mathbf{r})
$$

The developments leading to relation (42) are possible because the friction condition is convex explaining why we have restricted our study to such friction criterion.

\subsubsection{Non-associated sliding rule}

If the sliding rule is non-associated, prior to the addition of a vector orthogonal to the tangent plane, we need to apply a transformation to the tangential velocity in order to recover the normality of the slips to the friction cone in the plane $r_{n}=$ const (Fig. 7):

$$
\mathbb{Q}^{q}\left(-\dot{\mathbf{u}}_{t}\right) \in \partial \Psi_{K_{\mu}\left(r_{n}\right)}\left(\mathbf{r}_{t}\right)
$$

Next, we add the term " $-\left(\dot{u}_{n}+\left\|\mathbb{Q}^{q}\left(-\dot{\mathbf{u}}_{t}\right)\right\|_{q_{\mu}}^{*}\right)$ n" to the left-hand side of (43) to recover the normality with respect to the superelliptic Coulomb's cone:

$$
-\left(\mathbb{Q}^{q}\left(\dot{\mathbf{u}}_{t}\right)+\left(\dot{u}_{n}+\left\|\mathbb{Q}^{q}\left(-\dot{\mathbf{u}}_{t}\right)\right\|_{q_{\mu}}^{*}\right) \mathbf{n}\right) \in \partial \Psi_{K_{\mu}}(\mathbf{r})
$$

The norm applied to the transformed tangential vector is the norm dual to the one defining the friction condition. This point will appear more clearer in the next lines. The construction detailed above can be viewed as a mapping $\mathscr{T}$ from the velocity space $\mathscr{V}$ into itself (Fig. 8):

$$
\mathscr{T}: \mathscr{V} \mapsto \mathscr{V}:-\dot{\mathbf{u}} \mapsto-\left(\mathbb{Q}^{q}\left(\dot{\mathbf{u}}_{t}\right)+\left(\dot{u}_{n}+\left\|\mathbb{Q}^{q}\left(-\dot{\mathbf{u}}_{t}\right)\right\|_{q_{\mu}}^{*}\right) \mathbf{n}\right)
$$




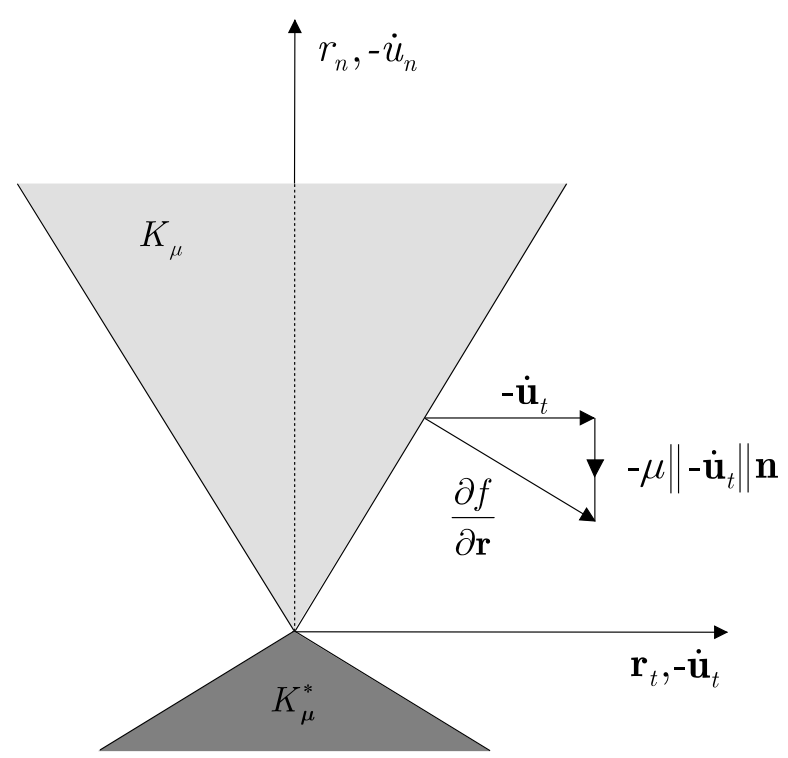

Fig. 7. Non-normality of the velocity vector.

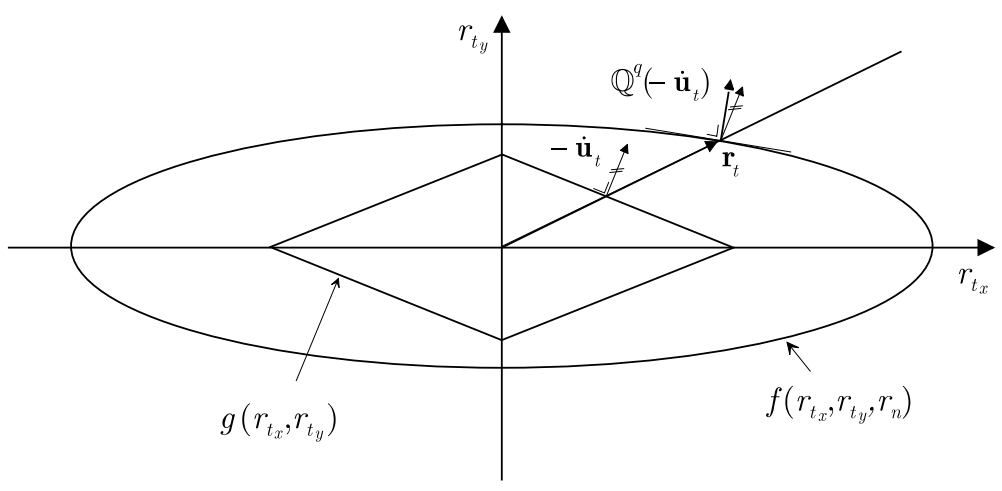

Fig. 8. Non-associated slip rule.

For the sake of clarity, let us denotes the transformed velocity vector by $\mathbf{v}$

$$
\mathbf{v}:=\mathscr{T}(-\dot{\mathbf{u}})
$$

and writes the frictional contact law (44) as

$$
\mathbf{v} \in \partial \Psi_{K_{\mu}}(\mathbf{r})
$$

This differential inclusion is equivalent to the convexity inequality

$$
\Psi_{K_{\mu}}\left(\mathbf{r}^{\prime}\right) \geqslant \Psi_{K_{\mu}}(\mathbf{r})+\mathbf{v} \cdot\left(\mathbf{r}^{\prime}-\mathbf{r}\right)
$$


which can be rewritten as

$$
\mathbf{r} \in K_{\mu}: \quad \mathbf{v} \cdot\left(\mathbf{r}^{\prime}-\mathbf{r}\right) \leqslant 0, \quad \forall \mathbf{r}^{\prime} \in K_{\mu}
$$

The relation (47) has been derived under the assumption of sliding and we need to show that this relation is also valid for the sticking and the separating cases. For $\mathbf{r}$ belonging to the interior of $K_{\mu}$, it is easy to see that the inequality (48) is satisfied only if the vector $\mathbf{v}$ is null. It is well-known from Convex Analysis [9] that the normal cone to a convex set is the null vector for elements belonging to its interior. The decomposition (1) being unique, the condition $\mathbf{v}=\mathbf{0}$ implied that

$$
\mathbf{v}_{t}=\mathbb{Q}^{q}\left(-\dot{\mathbf{u}}_{t}\right)=\mathbf{0} \quad \text { and } \quad v_{n}=-\left(\dot{u}_{n}+\left\|\mathbb{Q}^{q}\left(-\dot{\mathbf{u}}_{t}\right)\right\|_{q_{\mu}}^{*}\right)=0
$$

Therefore $\dot{\mathbf{u}}$ vanishes, the corresponding status is sticking. As a last step, we need to prove that the relation (47) will address the separating case. At this point $\mathbf{r}=\mathbf{0}$, the subdifferential of $\Psi_{K_{\mu}}$ coincides with the dual cone $K_{\mu}^{*}$

$$
\partial \Psi_{K_{\mu}}(\mathbf{0})=K_{\mu}^{*}
$$

defined by (9). As a consequence of the duality between the cones, we have at $\mathbf{v}=\mathbf{0}$,

$$
\partial \Psi_{K_{\mu}^{*}}(\mathbf{0})=K_{\mu}
$$

It is easy to show that if $\mathbf{r}=\mathbf{0}$, the condition $\mathbf{v} \in K_{\mu}^{*}$ corresponds to the separating case. In fact, by combining the definition of $\mathbf{v}(46)$ and the inequality

$$
\left\|\mathbf{v}_{t}\right\|_{q_{\mu}}^{*}+v_{n} \leqslant 0
$$

the impenetrability condition is recovered

$$
-\dot{u}_{n} \leqslant 0
$$

At this point, we understand that in (44) the norm can not be arbitrary and should be chosen properly in order to ensure that the frictional contact law encompasses the case of separation. In conclusion, the relation (47) describes the full frictional contact law for contacting bodies. Although this relation provides further insight into such complex frictional contact laws, additional developments can still be made to establish a relationship between $-\dot{\mathbf{u}}$ and $\mathbf{r}$ based on a normality rule. To do this, the relation $\mathbf{v} \in \partial \Psi_{K_{\mu}}(\mathbf{r})$ needs to be further developed. We first apply the Legendre-Fenchel transform to inverse the relation (47)

$$
\sup _{\mathbf{r}}\left[\mathbf{v} \cdot \mathbf{r}-\Psi_{K_{\mu}}(\mathbf{r})\right]=\sup _{\mathbf{r} \in K_{\mu}}\left[\mathbf{v}_{t} \cdot \mathbf{r}_{t}+v_{n} r_{n}\right]
$$

The scalar product in (54) satisfies the following inequalities

$$
\begin{aligned}
\mathbf{v} \cdot \mathbf{r}= & v_{t_{x}} r_{t_{x}}+v_{t_{y}} r_{t_{y}}+v_{n} r_{n} \leqslant\left|v_{t_{x}}\right|\left|r_{t_{x}}\right|+\left|v_{t_{y}}\right|\left|r_{t_{y}}\right|+v_{n} r_{n} \leqslant\left|\mu_{x} v_{t_{x}}\right|\left|\frac{r_{t_{x}}}{\mu_{x}}\right|+\left|\mu_{y} v_{t_{y}}\right|\left|\frac{r_{t_{x}}}{\mu_{x}}\right| \\
& +v_{n} r_{n} \leqslant\left\|\mathbf{v}_{t}\right\|_{q_{\mu}}^{*}\left\|\mathbf{r}_{t}\right\|_{q_{\mu}}+v_{n} r_{n}
\end{aligned}
$$


where the Hölder inequality has been used in the last step. The the norm $\left\|\mathbf{r}_{t}\right\|_{q_{\mu}}$ is bounded by $r_{n}$

$$
\mathbf{v}_{t} \cdot \mathbf{r}_{t} \leqslant r_{n}\left\|\mathbf{v}_{t}\right\|_{q_{\mu}}^{*}
$$

The friction force has been eliminated and the supremum is calculated according to

$$
\sup _{r_{n} \in \mathbb{R}_{+}}\left[\left(\left\|\mathbf{v}_{t}\right\|_{q_{\mu}}^{*}+v_{n}\right) r_{n}\right]
$$

Two distinct possibilities emerge: if $\left\|\mathbf{v}_{t}\right\|_{q_{u}}^{*}+v_{n} \leqslant 0$ then the supremum (55) is 0 since $r_{n} \geqslant 0$; if on the other hand $\left\|\mathbf{v}_{t}\right\|_{q_{\mu}}^{*}+v_{n} \geqslant 0$ then since the value of $r_{n}$ is unbounded, so is the supremum (55). Thus, we have

$$
\sup _{r_{n} \in \mathbb{R}_{+}}\left[\left(\left\|\mathbf{v}_{t}\right\|_{q_{\mu}}^{*}+v_{n}\right) r_{n}\right]=\Psi_{K_{\mu}^{*}}(\mathbf{v})
$$

and the inverse law is

$$
\mathbf{r} \in \partial \Psi_{K_{\mu}^{*}}(\mathbf{v})
$$

The functions $\Psi_{K_{\mu}^{*}}(\mathbf{v})$ and $\Psi_{K_{\mu}}(\mathbf{r})$ satisfy the following relation:

$$
\Psi_{K_{\mu}^{*}}\left(\mathbf{v}^{\prime}\right)+\Psi_{K_{\mu}}\left(\mathbf{r}^{\prime}\right) \geqslant \mathbf{v}^{\prime} \cdot \mathbf{r}^{\prime}, \quad \forall\left(\mathbf{v}^{\prime}, \mathbf{r}^{\prime}\right) \in \mathscr{V} \times \mathscr{F}
$$

A pair $(\mathbf{v}, \mathbf{r})$ related by the frictional contact law satisfies

$$
\mathbf{v} \in \partial \Psi_{K_{\mu}}(\mathbf{r}) \Longleftrightarrow \mathbf{r} \in \partial \Psi_{K_{\mu}^{*}}(\mathbf{v}) \Longleftrightarrow \Psi_{K_{\mu}^{*}}(\mathbf{v})+\Psi_{K_{\mu}}(\mathbf{r})=\mathbf{v} \cdot \mathbf{r}
$$

To recover a relation between the dual variables $-\dot{\mathbf{u}}$ and $\mathbf{r}$, we add $\mathbf{r}^{\prime} \cdot\left(-\dot{\mathbf{u}}^{\prime}\right)$ to both sides of (58),

$$
\left\{\begin{array}{l}
\forall\left(\mathscr{T}\left(-\dot{\mathbf{u}}^{\prime}\right), \mathbf{r}^{\prime}\right) \in \mathscr{V} \times \mathscr{F}: \\
\Psi_{K_{\mu}^{*}}\left(\mathscr{T}\left(-\dot{\mathbf{u}}^{\prime}\right)\right)+\Psi_{K_{\mu}}\left(\mathbf{r}^{\prime}\right)+\mathbf{r}^{\prime} \cdot\left(\left(-\dot{\mathbf{u}}^{\prime}\right)-\mathscr{T}\left(-\dot{\mathbf{u}}^{\prime}\right)\right) \geqslant \mathbf{r}^{\prime} \cdot\left(-\dot{\mathbf{u}}^{\prime}\right)
\end{array}\right.
$$

The left-hand side of (59) is a function of both $-\dot{\mathbf{u}}^{\prime}$ and $\mathbf{r}^{\prime}$, which cannot be separated in the sum of two functions, one of $-\dot{\mathbf{u}}^{\prime}$ and another of $\mathbf{r}^{\prime}$. We call this function a bi-potential and its general expression is

$$
b_{\mathrm{c}}\left(-\dot{\mathbf{u}}^{\prime}, \mathbf{r}^{\prime}\right):=\Psi_{K_{\mu}^{*}}\left(\mathscr{T}\left(-\dot{\mathbf{u}}^{\prime}\right)\right)+\Psi_{K_{\mu}}\left(\mathbf{r}^{\prime}\right)+\mathbf{r}^{\prime} \cdot\left(\left(-\dot{\mathbf{u}}^{\prime}\right)-\mathscr{T}\left(-\dot{\mathbf{u}}^{\prime}\right)\right)
$$

By developing the right-hand side of (60) and taking into account the equivalence

$$
\Psi_{K_{\mu}^{*}}(\mathscr{T}(-\dot{\mathbf{u}})) \Longleftrightarrow \Psi_{\mathbb{R}_{-}}\left(-\dot{u}_{n}\right)
$$


we obtain the bi-potential for the present frictional contact model:

$$
b_{\mathrm{c}}(-\dot{\mathbf{u}}, \mathbf{r})=\Psi_{K_{\mu}}(\mathbf{r})+\Psi_{\mathbb{R}_{-}}\left(-\dot{u}_{n}\right)+\left(\mathbb{\square}-\mathbb{Q}^{q}\right) \cdot \mathbf{r}_{t}+r_{n}\left\|\mathbb{Q}^{q}\left(-\dot{\mathbf{u}}_{t}\right)\right\|_{q_{\mu}}^{*}
$$

The bi-potential satisfies the fundamental inequality

$$
b_{\mathrm{c}}\left(-\dot{\mathbf{u}}^{\prime}, \mathbf{r}^{\prime}\right) \geqslant-\dot{\mathbf{u}}^{\prime} \cdot \mathbf{r}^{\prime}
$$

The equality is reached in (63) for an extremal pair that is a pair $(-\dot{\mathbf{u}}, \mathbf{r})$ related by the frictional contact law:

$$
b_{\mathrm{c}}(-\dot{\mathbf{u}}, \mathbf{r})=-\dot{\mathbf{u}} \cdot \mathbf{r}
$$

The relations (63) and (64) can be combined to give

$$
\begin{aligned}
& \forall \mathbf{r}^{\prime} \in \mathscr{F}: \quad b_{\mathrm{c}}\left(-\dot{\mathbf{u}}, \mathbf{r}^{\prime}\right)-b_{\mathrm{c}}(-\dot{\mathbf{u}}, \mathbf{r}) \geqslant-\dot{\mathbf{u}} \cdot\left(\mathbf{r}^{\prime}-\mathbf{r}\right) \\
& \forall-\dot{\mathbf{u}}^{\prime} \in \mathscr{V}: \quad b_{\mathrm{c}}\left(-\dot{\mathbf{u}}^{\prime}, \mathbf{r}\right)-b_{\mathrm{c}}(-\dot{\mathbf{u}}, \mathbf{r}) \geqslant \mathbf{r} \cdot\left(\left(-\dot{\mathbf{u}}^{\prime}\right)-(-\dot{\mathbf{u}})\right)
\end{aligned}
$$

which means that

- the bi-potential is bi-convex that is $b_{\mathrm{c}}(-\dot{\mathbf{u}}, \mathbf{r})$ is a convex function of $-\dot{\mathbf{u}} \in \mathscr{V}$ for each $\mathbf{r} \in \mathscr{F}$ and a convex function of $\mathbf{r} \in \mathscr{F}$ for each $-\dot{\mathbf{u}} \in \mathscr{V}$

- the flow rule and its inverse derive from the bi-potential $b_{\mathfrak{c}}(-\dot{\mathbf{u}}, \mathbf{r})$

$$
-\dot{\mathbf{u}} \in \partial_{\mathbf{r}} b_{\mathrm{c}}(-\dot{\mathbf{u}}, \mathbf{r}) \text { and } \quad \mathbf{r} \in \partial_{-\dot{\mathbf{u}}} b_{\mathrm{c}}(-\dot{\mathbf{u}}, \mathbf{r})
$$

The relations (67) are essentials for the derivation of stationary principles involving a functional that depends now on both the velocities and the stress $[2,3]$.

\section{Conclusion}

Recent theoretical work [7] on orthotropic friction shows that an accurate description of this phenomena involves limit surfaces of complex geometry. Furthermore, the sliding rule may deviate significantly from the normality. An appropriate way to describe these models in a unifying way is to use superelliptic friction conditions. With this type of curve a large number of practical situations can be accommodated by simply adapting the roundedness factor that governs the shape of the superellipse. To generate a non-associated sliding rule, we chose the same kind of superellipse but with a different semi-axes ratio. The governing equations of such models are rather complex and a more enlightening formulation is provided. Indeed, the weak variational structure of these models has been exhibited. The normality rule has been recovered but only in a implicit form. This formulation will be used in forthcoming papers to derive a robust algorithm and therefore solve practical problems involving such models. 


\section{Appendix A. Hölder inequality for finite sequences}

Consider the space $\mathbb{R}^{n}$ and define

$$
\left.\|\mathbf{x}\|_{p}=\sum_{i=1}^{n}\left|x_{i}\right|^{p}\right)^{\frac{1}{p}} \text { with } 1 \leqslant p \leqslant \infty
$$

It follows immediately from the previous definition that $\|\bullet\|_{p}$ satisfies the first axioms of a norm, i.e.

- $\|\mathbf{x}\|_{p}=0$ if and only if $\mathbf{x}=\mathbf{0}$,

- $\|\lambda \mathbf{x}\|_{p}=\lambda\|\mathbf{x}\|_{p}, \forall \lambda>0, \forall \mathbf{x} \in \mathbb{R}^{n}$.

Consider two vectors $\mathbf{x}$ and $\mathbf{y}$ belonging to $\mathbb{R}^{n}$, the Hölder inequality asserts that

$$
\left|\sum_{i=1}^{n} x_{i} y_{i}\right| \leqslant \sum_{i=1}^{n}\left|x_{i}\right|\left|y_{i}\right| \leqslant\|\mathbf{x}\|_{p}\|\mathbf{y}\|_{q}
$$

where $p, q \in\left[1, \infty\left[\right.\right.$ and $\frac{1}{p}+\frac{1}{q}=1$.

\section{References}

[1] P. Alart, A. Curnier, A mixed formulation for frictional contact problems prone to Newton-like solution methods, Computer Methods in Applied Mechanics and Engineering 92 (1991) 353-375.

[2] G. de Saxcé, Z.-Q. Feng, The bi-potential method: a constructive approach to design the complete contact law with friction and improved numerical algorithms, Mathematical and Computer Modelling 28 (4-8) (1998) 225-245.

[3] G. de Saxcé, Z.-Q. Feng, New inequality and functional for contact friction: the implicit standard material approach, Mechanics of Structures and Machines 19 (1991) 301-325, 12. 6.

[4] B. Halphen, Nguyen Quoc Son, Sur les matériaux standard généralizés, Journal de Mécanique 14 (1975) 39-63, 10. 9.

[5] R. Michałowski, Z. Mróz, Associated and non-associated sliding rules in contact friction problems, Archives of Mechanics 30 (1978) 259-276.

[6] J.-J. Moreau, Sur les lois de frottement, de plasticité et de viscosité, Comptes Rendus de l'Académie des Sciences 271 (1970) 608-611.

[7] Z. Mróz, S. Stupkiewicz, An anisotropic fricition and wear model, International Journal of Solids and Structures 31 (1994) 1113-1131.

[8] Nguyen Quoc Son, Matériaux élasto-visco-plastique et élastoplastique à potentiel généralisé, Comptes Rendus de l’Académie des Sciences 277 (1973) 915-918.

[9] R.T. Rockafellar, J.-B. Wets, Variational Analysis, Springer, Berlin, 1998. 